\title{
FGFR2 mutations in Pfeiffer syndrome
}

Sir-In the past few months, several genetic diseases have been ascribed to mutations in genes of the fibroblast growth factor receptor (FGFR) family, including achondroplasia $(F G F R 3)^{1-2}$, Crouzon syndrome $(F G F R 2)^{3}$ and Pfeiffer syndrome $(F G F R 1)^{4}$. In addition, two clinically distinct craniosynostotic conditions, JacksonWeiss and Crouzon syndromes, have been ascribed to allelic mutations in the FGFR2 gene, suggesting that FGFR2mutations might havevariable phenotypic effects 5 . We have recently found point mutations in the FGFR2 gene in two unrelated cases of Pfeiffer syndrome supporting the view that this craniosynostotic syndrome, is a genetically heterogenous condition ${ }^{4,6}$.

Pfeiffer syndrome is an autosomal dominant form of acrocephalosyndactyly (ACS) characterized by craniosynostosis (brachycephaly type) with deviation and enlargement of the thumbs and great toes, brachymesophalangy, with phalangeal ankylosis and a varying degree of soft tissue syndactyly ${ }^{7,8}$. One of our sporadic cases and one familial form of ACS fulfilled the clinical criteria of the Pfeiffer syndrome, with particular respect to interphalangeal ankylosis (Fig. 1). There is no confusion with Crouzon syndrome, in which no hand anomalies and occasional radio-ulnar synostoses have been reported, or Jackson-Weiss syndrome, which includes a tarsalmetatarsal coalescence and a medial deviation of broad great toes.

Using a combination of single strand conformation polymorphism and sequence analyses of amplified exons in a case of sporadic Pfeiffer syndrome, we found an $A$ to $G$ transition in the $3^{\prime}$ acceptor splice site of the intron adjacent to exon $B$ of FGFR2, disrupting the consensus sequence required for the normal processing of the RNA transcript (Fig. 1). Whether this mutation results in an aberrant protein or in functional haploinsufficiency for the exon Bcontaining splice variant is under investigation.

In a familial form of Pfeiffer syndrome, we found an $\mathrm{A}$ to $\mathrm{C}$ transition at nucleotide 974 (codon 321 ) of the coding sequence (exon B), creating an Eael site and changing
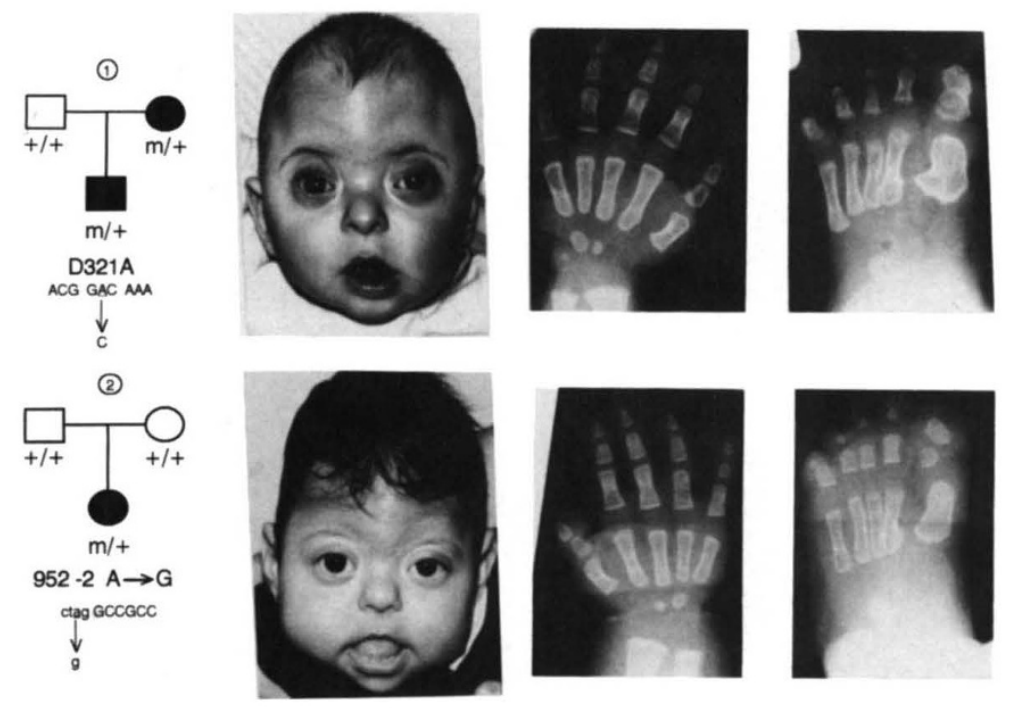

Fig. 1 Mutations of FGFR2 in two unrelated patients with craniofacial, hand and foot anomalies characteristic of Pfeiffer syndrome. Note midface retrusion, hypertelorism, proptosis and radiological evidence of enlargement of thumb, with ankylosis of the second and third phalanges and the short, broad and deviated great toes. $m$, mutation; +, wild type sequence. The sequence of wild type and mutant genes are shown and the arrow indicates the base substitution resulting in the mutation.

an aspartic acid into an alanine in the third immunoglobulin-like domain of the protein (Fig. 1). These base substitutions were not found in 80 normal controls and the recurrent mutation in FGFR1 previously reported in Pfeiffer syndrome ${ }^{4}$ was not found in our patients (not shown).

Muenke et al. ${ }^{4}$ emphasised the genetic heterogeneity of Pfeiffer syndrome, as not all familial forms of the disease were linked to the region of chromosome 8 where FGFR 1 maps ${ }^{6}$. The discovery of mutations in the extracellular domain of FGFR2 in unrelated cases of Pfeiffer syndrome suggests that this condition can be also accounted for by mutations in FGFR2. Finally, our observation gives strong support to the variable clinical expression of FGFR2 mutations in man, which, with additional examples described on page 165 of this issue', now account for four clinically distinct craniosynostotic syndromes: Apert ${ }^{9}$, Crouzon $^{3}$, Jackson-Weiss ${ }^{5}$ and Pfeiffer syndromes.
Elisabeth Lajeunie Hong Wei Ma Jacky Bonaventure Arnold Munnich Martine Le Merrer Unité de Recherches sur les Handicaps Génétiques de l'Enfant, INSERM U. 393, Hôpital des Enfants Malades, 149 rue de Sèvres, 75743 Paris Cedex 15, France

Dominique Renier

Département de Neurochirurgie, Hôpital des Enfants Malades, 149 rue de Sèvres, 75743 Paris Cedex 15, France

1. Shiang, R. et al. Cell 78, 335-342 (1994).

2. Rousseau, F. ot al. Nature $371,252-254$ (1994).

3. Reardon, W. et al. Nature Genet. 8, 98-103 (1994)

4. Muenke, M. et al. Nature Genet. 8, 269-274 (1994).

5. Jabs, E.W. et al. Nature Genet. 8, 275-279 (1994).

6. Robin, N.H. et al. Am. J. hum. Genet. 55, A1171 (1994).

7. Pfeiffer, R.A. Z. Kinderheilk 90, 301-320 (1964).

8. Cohen, M.M. Jr. Am. J. med. Genet. 45, 300307 (1993).

9. Wilkie, A.O.M. et al. Nature Genet. 9, 165-172 (1995). 